\title{
DNA Barcoding and Molecular Phylogeny of Drosophila lini and Its Sibling Species
}

\author{
Yi-Feng Li, ${ }^{1}$ Shuo-Yang Wen, ${ }^{1}$ Kuniko Kawai, ${ }^{2}$ Jian-Jun Gao, ${ }^{3}$ Yao-Guang Hu, ${ }^{2}$ \\ Ryoko Segawa, ${ }^{4}$ and Masanori J. Toda ${ }^{2,5}$ \\ ${ }^{1}$ Department of Entomology, South China Agricultural University, Guangzhou 510642, China \\ ${ }^{2}$ Institute of Low Temperature Science, Hokkaido University, Sapporo, Hokkaido 060-0819, Japan \\ ${ }^{3}$ Laboratory for Conservation and Utilization of Bio-resources, Yunnan University, Kunming 650091, China \\ ${ }^{4}$ Evolutionary Genetics Laboratory, Department of Biology, Tokyo Metropolitan University, Tokyo 192-03, Japan \\ ${ }^{5}$ The Hokkaido University Museum, Hokkaido University, Sapporo 060-0810, Japan \\ Correspondence should be addressed to Masanori J. Toda, hutian@pop.lowtem.hokudai.ac.jp
}

Received 15 July 2011; Accepted 27 October 2011

Academic Editor: Artyom Kopp

Copyright ( 2012 Yi-Feng Li et al. This is an open access article distributed under the Creative Commons Attribution License, which permits unrestricted use, distribution, and reproduction in any medium, provided the original work is properly cited.

Drosophila lini and its two sibling species, D. ohnishii and D. ogumai, are hardly distinguishable from one another in morphology. These species are more or less reproductively isolated. The mitochondrial ND2 and COI-COII and the nuclear ITS1-ITS2 regions were sequenced to seek for the possibility of DNA barcoding and to reconstruct the phylogeny of them. The character-based approach for DNA barcoding detected some diagnostic nucleotides only for monophyletic D. ogumai, but no informative sites for the other two very closely species, D. lini and D. ohnishii, of which strains intermingled in the molecular phylogenetic trees. Thus, this study provides another case of limited applicability of DNA barcoding in species delineation, as in other cases of related Drosophila species. The molecular phylogenetic tree inferred from the concatenated sequences strongly supported the monophyly of the cluster of the three species, that is, the lini clade. We propose some hypotheses of evolutionary events in this clade.

\section{Introduction}

Studies of just diverging populations or species shed light on speciation mechanisms. An important evolutionary process in speciation is the diversification of genes between populations. Most comprehensive information on gene (DNA) evolution associated with speciation has been accumulated for the Drosophila melanogaster species subgroup, especially the D. simulans clade (e.g., [1-6]), and the D. obscura species group $[7,8]$. In comparison, speciation mechanisms have been less explored in the $D$. montium species subgroup, in spite of its highest species diversity $(=89)$ [9] in the melanogaster group, with a variety of species at different stages of speciation process. On the other hand, molecular markers have been used to detect cryptic species under incipient speciation process. In the D. montium subgroup as well, such a molecular approach has recently been employed to reveal the presence of a cryptic species in the D. serrata species complex [10]. "DNA barcoding" is proposed as a promising tool not only for rapid identification of known species, that is, "species identification," but also for discovery and delimitation of species, that is, "species discovery" or "DNA taxonomy" [11-13].

Discovery of a sibling species of Drosophila lini is one of cases in which molecular characters were used for "species discovery" in early days. It was first recognized as a species closely related to but different from $D$. lini based on the results of electrophoretic analyses [14]. Then, the studied "D. lini-like" strain, MMY326, from Pyinoolwin in central Myanmar, along with another strain (MMY307) from the same locality, was described as D. ohnishii [15]. At the same time, another sibling species, D. ogumai, was described for two strains (RGN3 and RGN206) from southern Myanmar [15]. It is, however, hard to morphologically distinguish among the three species, D. lini, D. ohnishii, and D. ogumai, especially between the former two, although $80-100 \%$ correct classification was achieved for them by discriminant analyses using 13 or 15 quantitative characters [15]. The 
evidence from cross-tests supports the presence of three sibling species. More or less strong postmating isolation is present among them: no F1 hybrids could be obtained from crosses between D. ogumai and D. ohnishii, while the other interspecific crosses produced fertile hybrid females but sterile males [16]. In addition, strong premating isolation was detected between $D$. ohnishii and D. lini or between $D$. ohnishii and D. ogumai, but not between D. lini and D. ogumai [16-18]. When the D. kikkawai species complex was established in the D. montium species subgroup of the D. melanogaster species group, D. lini was included in it [19]. Subsequent molecular phylogenetic studies consistently supported the close relationships between D. lini (and its siblings) and D. kikkawai (and its siblings, D. bocki and D. leontia) [14, 20-24]. However, the relationships between D. lini and its siblings have not been resolved yet.

Up to date, it is known that D. ohnishii is distributed in central Myanmar to southwestern China (Xishuangbanna), just occupying the intermediate range between the ranges of the two allopatric species, $D$. lini distributed in southern China to Taiwan and D. ogumai in southern Myanmar [18]. Thus, the premating isolation is seen between the parapatric neighbors of the three species. Based on this biogeographical evidence, a hypothesis that the premating isolation has evolved through the process of reinforcement in the secondary contact zone between $D$. ohnishii and either neighboring species has been proposed [18]. To test or refine this hypothesis, the present study aims at revealing reliable phylogenetic relationships among these three species based on DNA sequence data. In addition, molecular diagnostics are searched to apply the DNA barcoding "species identification" to these sibling species that are hard to be distinguished morphologically from one another.

When focusing on very closely related species, one should select rapidly evolving regions, for example, mitochondrial genes [25] or nuclear rDNA internal transcribed spacer (ITS) [26], as markers. The mitochondrial cytochrome $c$ oxidase subunit I (COI) gene has been widely used as DNA barcoding for "species identification": its 648-base pair (bp) fragment is the standard marker in the Barcode of Life project $[11,12]$. In the present study, we employed two mitochondrial loci, $\mathrm{NADH}$ dehydrogenase subunit 2 (ND2) and cytochrome $c$ oxidase subunit I and II (COI-COII), and one nuclear locus, rDNA internal transcribed spacer 1 and 2 (ITS1-ITS2), to examine the phylogenetic relationships among isofemale strains of $D$. lini and its sibling species and to find possible molecular diagnostics for each species of them.

\section{Materials and Methods}

Seven isofemale strains of $D$. lini, four strains of D. ohnishii, and two strains of D. ogumai were used as focal OTUs, and one strain each of D. kikkawai, D. bocki, D. leontia, and D. barbarae of the kikkawai complex, and one strain each of D. jambulina and D. seguyi belonging to the montium subgroup were added as ingroup OTUs (Table 1). Three of these isofemale strains (MLN24 and MLN45 of D. ohnishii and MLN260 of D. barbarae) were established in 2003 from
TABLE 1: List of experimental strains.

\begin{tabular}{lcc}
\hline Species & Lines & Collection locality \\
\hline & 3146.1 & Taiwan, China \\
& DHS315 & Dinghushan, Guangdong, China \\
& DHS410 & Dinghushan, Guangdong, China \\
& DHS501 & Dinghushan, Guangdong, China \\
D. lini & NKS9212 & Nankunshan, Guangdong, China \\
& NKS9231 & Nankunshan, Guangdong, China \\
& NK9242 & Nankunshan, Guangdong, China \\
\hline & MMY309 & Pyinoolwin, Myanmar \\
D. ohnishii & MMY326 & Pyinoolwin, Myanmar \\
& MLN24 & Menglun, Yunnan, China \\
& MLN45 & Menglun, Yunnan, China \\
\hline D. ogumai & RGN3 & Yangon, Myanmar \\
\hline D. bocki & Y163 & Yangon, Myanmar \\
D. leontia & AO-2 & ? \\
D. kikkawai & OGS4 & ? \\
D. barbarae & MLN260 & Menglun, Yunnan, China \\
D. jambulina & NH115 & ? \\
D. seguyi & K2 & $?$ \\
\hline
\end{tabular}

TABLE 2: Target regions and primer sequences in the present study.

\begin{tabular}{lcc}
\hline Target region & Primer sequence $\left(5^{\prime}-3^{\prime}\right)$ & Length $(\mathrm{bp})$ \\
\hline $\begin{array}{l}\text { Mitochondrial loci } \\
\text { ND2 }\end{array}$ & $\begin{array}{c}\text { AAGCTACTGGGTTCATACC } \\
\text { ATATTTACAGCTTTGAAGG }\end{array}$ & 926 \\
COI-COII & $\begin{array}{c}\text { ATACCTCGACG(AT)TATTGA } \\
\text { GTTTAAGAAACCAGTACTTG }\end{array}$ & 842 \\
\hline $\begin{array}{l}\text { Nuclear locus } \\
\text { ITS1-ITS2 }\end{array}$ & $\begin{array}{l}\text { TCCGTAGGTGAACCTGCGG } \\
\text { GTTAGTTTCTTTTCCTC }\end{array}$ \\
\hline Total & & \\
\hline
\end{tabular}

Menglun, southern part of Yunnan Province, China, but all the others derived from the stocks of Tokyo Metropolitan University and have been maintained in laboratory on cornmeal-malt medium at $23^{\circ} \mathrm{C}$ under continuous light for more than 12 years. The species status of the closely related species, that is, D. lini/D. ohnishii/D. ogumai and D. kikkawai/D. bocki/D. leontia, was confirmed by cross-tests in previous studies $[16,27]$.

Total DNA was extracted from a single fly using a rapid method [28]. The target regions (Table 2) were amplified on an iCycler Thermal Cycler (Bio-Rad) with the PCR cycle program comprised a $5 \mathrm{~min}$ of predenaturation at $94^{\circ} \mathrm{C}, 35$ cycles of amplification ( $1 \mathrm{~min}$ of denaturing at $94^{\circ} \mathrm{C} ; 1 \mathrm{~min}$ of annealing at $53^{\circ} \mathrm{C}$ for $\mathrm{COI}-\mathrm{II}$ and $\mathrm{ND} 2,56^{\circ} \mathrm{C}$ for ITS1ITS2, $1 \mathrm{~min}$ of extension at $72^{\circ} \mathrm{C}$ ), and final extension at $72^{\circ} \mathrm{C}$ for $5 \mathrm{~min}$. The amplicons were purified by precipitation with isopropanol and then subjected to sequencing reaction using BigDye Terminator v3.1 Cycle Sequencing Kit (Applied Biosystems) following the recommended protocol. 


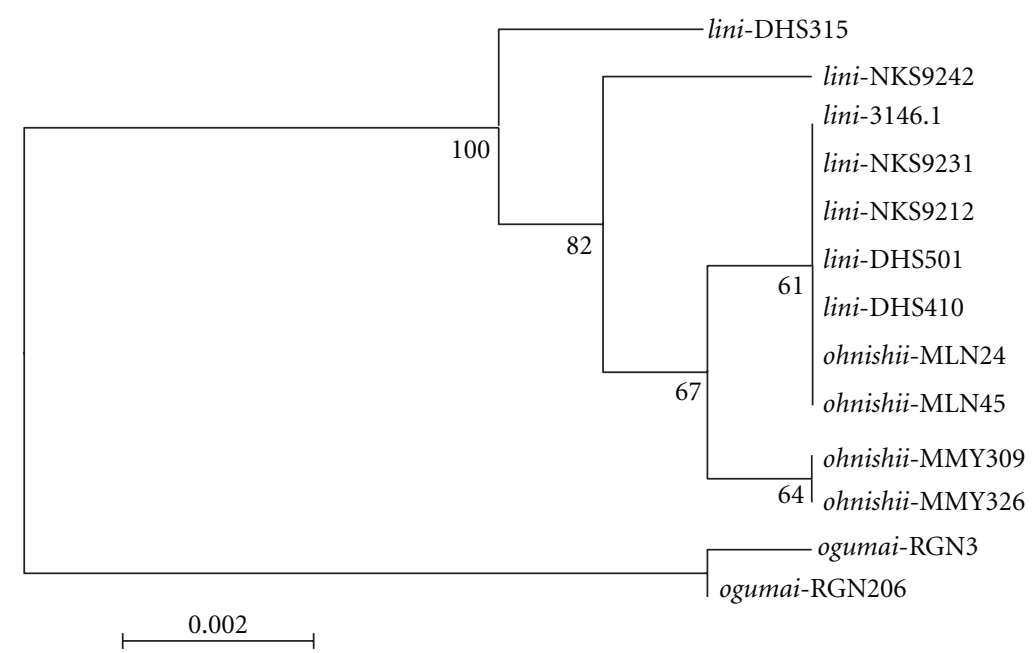

Figure 1: Neighbor-joining (NJ) tree inferred from ND2 sequences of 13 strains of D. lini and its sibling species (D. ohnishii and D. ogumai). Numbers below branches indicate the bootstrap percentages.

The sequences were analyzed on the 3100-Avant Genetic Analyzer (Applied Biosystems).

The ITS1-ITS2 sequences of three species of the montium subgroup were downloaded from GenBank, AY278412 for D. barbarae; AY278419 for D. jambulina, and AY278431 for D. seguyi. For the three species of the D. melanogaster subgroup employed as outgroups, the corresponding sequences were also downloaded from GenBank: AF200829 for ND2 and COI-COII and M21017 for ITS1-ITS2 of D. melanogaster; AF200846 for ND2 and COI-COII and Z28413 for ITS1-ITS2 of D. simulans; AF200831 for ND2 and COI-COII, Z28538 for ITS1-ITS2 of D. mauritiana.

DNA sequences were edited and analyzed using MEGA 5.05 [29]. Phylogenetic trees were constructed by the Neighbor-Joining (NJ) method with bootstrap test (1000 replicates) using the Kimura 2-parameter model, with gaps treated by pairwise deletion. For searching DNA barcoding diagnostics, we focused only on the three sibling species with multiple test strains, and applied both of tree- and characterbased methods to each of different loci separately. We used the phylogeny-based approach in the former method, examining the monophyly of each species on a phylogenetic tree [30-33]. The character-based method identifies a set of diagnostic nucleotides in the DNA barcode sequence: the four standard nucleotides $(A, T, C, G)$ if found in fixed states in one species can be used as simple pure diagnostics for identifying that species [34]. To examine molecular genealogies for the focal OTUs, we constructed an NJ tree based on the concatenated sequences of the three loci, and applied an estimated divergence time, 5.4 million years ago (Mya) [35], between $D$. melanogaster and $D$. simulans as a calibration point to estimate the divergence time of each node. Before the analysis using the concatenated sequence data, we conducted a Bayesian concordance analysis to test the concordance among the three regions, that is, ND2, COI-COII, and ITS1-ITS2, using BUCKy [36]. The DNA sequences of each region were analyzed using MrBayes 3.1.2 [37] for Bayesian phylogenetic estimation. Firstly, phylogenetic trees were constructed for each region via the Markov chain Monte Carlo (MCMC) method (number of generations for runs $=1,000,000$, nucleotide substitution model $=$ GTR (general time-reversible)), and then, the output of MrBayes was summarized using the mbsum program of BUCKy, and the primary concordance tree was generated with sample-wide concordance factors using default setting in BUCKy.

\section{Results}

3.1. DNA Barcoding for D. lini and Its Sibling Species. We sequenced the ND2 gene in D. lini and its siblings and some other species of the montium subgroup. The whole sequence of this gene is $1206 \mathrm{bp}$ in most species of the D. obscura species group [38]. Our obtained sequences covered most of this region (from the site 34 to 959). The alignment of the sequences included no indel. The GenBank accession numbers of these sequences are AY739939-AY739956. The NJ tree for 13 strains of $D$. lini and its siblings showed that D. ogumai was monophyletic but that D. lini and D. ohnishii were nonmonophyletic with overlap of strains of these two species (Figure 1). There were 20 informative sites in the aligned 13 sequences of $D$. lini and its siblings. Of these sites, 11 nucleotides were specific to D. ogumai, and thus can be used as diagnostic nucleotides for identification of this species among the siblings (Table 3 ). However, there was no speciesspecific, fixed nucleotide for either D. lini or D. ohnishii.

The whole COI and COII sequences are 1536 and $684 \mathrm{bp}$, respectively, in D. yakuba [39]. The COI-COII region we sequenced covered $130 \mathrm{bp}$ of COI and $639 \mathrm{bp}$ of COII. The GenBank accession numbers of these sequences are AY737604AY737622. The NJ tree based on the COI-COII sequences of the 13 strains of $D$. lini and its siblings showed the monophyly of D. ogumai but nonmonophyly for either $D$. lini or D. ohnishii (Figure 2). Twelve informative sites were detected from this region, among which five were species-specific, diagnostic nucleotides for D. ogumai (Table 4). The 


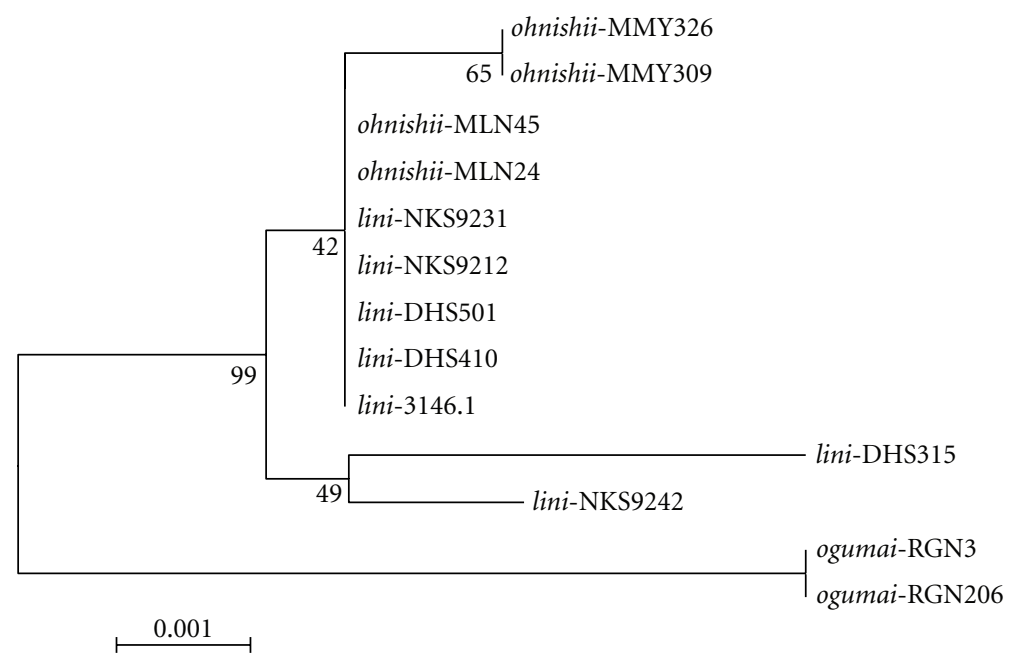

Figure 2: Neighbor-joining (NJ) tree inferred from COI-COII sequences of 13 strains of $D$. lini and its sibling species (D. ohnishii, and D. ogumai). Numbers below branches indicate the bootstrap percentages.

TAble 3: Nucleotides at 20 informative sites in ND2 sequences of 13 strains of D. lini and its sibling species (D. ohnishii and D. ogumai). Diagnostic nucleotides for DNA barcoding are indicated with an asterisk. $N$ is the number of strains sequenced. The positions of nucleotide sites are based on the sequence of D. obscura [38]. Polymorphic sites are shown with code letters R (A/G,), Y (T/C), S (C/G), W (A/T), and $\mathrm{M}(\mathrm{A} / \mathrm{C})$.

\begin{tabular}{|c|c|c|c|c|c|c|c|c|c|c|c|c|c|c|c|c|c|c|c|c|c|c|}
\hline \multicolumn{23}{|c|}{ Position } \\
\hline \multirow{4}{*}{ Species } & $N$ & & & & & & & & & $N D$ & (si & es 34 & -959 & & & & & & & & & \multirow{4}{*}{ Phylogeny } \\
\hline & & 1 & 2 & 3 & 3 & 4 & 4 & 4 & 4 & 5 & 5 & 6 & 6 & 7 & 7 & 7 & 8 & 8 & 9 & 9 & 9 & \\
\hline & & 1 & 5 & 4 & 4 & 0 & 1 & 3 & 8 & 4 & 5 & 6 & 6 & 4 & 8 & 9 & 2 & 9 & 0 & 3 & 5 & \\
\hline & & 4 & 5 & 2 & 5 & 8 & 1 & 4 & 7 & 3 & 2 & 7 & 9 & 7 & 1 & 6 & 9 & 5 & 0 & 4 & 4 & \\
\hline D. lini & 7 & $\mathrm{R}$ & $\mathrm{T}$ & $\mathrm{C}$ & A & $\mathrm{Y}$ & $\mathrm{A}$ & $\mathrm{G}$ & $\mathrm{S}$ & $\mathrm{T}$ & $\mathrm{A}$ & W & $\mathrm{W}$ & $\mathrm{R}$ & $\mathrm{T}$ & $\mathrm{C}$ & $\mathrm{M}$ & $\mathrm{C}$ & $\mathrm{T}$ & $\mathrm{C}$ & $\mathrm{C}$ & Non-monophyletic \\
\hline D. ohnishii & 4 & A & $\mathrm{T}$ & $\mathrm{C}$ & A & $\mathrm{C}$ & A & G & $\mathrm{S}$ & $\mathrm{T}$ & $\mathrm{R}$ & A & A & G & $\mathrm{T}$ & $\mathrm{C}$ & $\mathrm{A}$ & $\mathrm{C}$ & $\mathrm{T}$ & $\mathrm{C}$ & $\mathrm{C}$ & Non-monophyletic \\
\hline D. ogumai & 2 & G & $\mathrm{C}^{*}$ & $\mathrm{~T}^{*}$ & $\mathrm{R}$ & $\mathrm{T}$ & $\mathrm{G}^{*}$ & $\mathrm{~A}^{*}$ & G & $\mathrm{C}^{*}$ & A & A & A & G & $\mathrm{C}^{*}$ & $\mathrm{~T}^{*}$ & $\mathrm{~A}$ & $\mathrm{~T}^{*}$ & $\mathrm{C}^{*}$ & $\mathrm{~T}^{*}$ & $\mathrm{~T}^{*}$ & Monophyletic \\
\hline
\end{tabular}

TABle 4: Nucleotides at 12 informative sites in COI-COII sequences of 13 strains of D. lini and its sibling species (D. ohnishii and D. ogumai). Diagnostic nucleotides for DNA barcoding are indicated with an asterisk. The positions of nucleotide sites are based on the whole length of the COI (1536 bp) and COII (684 bp) sequences of D. yakuba [39]. See Table 3 for further explanations.

\begin{tabular}{|c|c|c|c|c|c|c|c|c|c|c|c|c|c|c|}
\hline \multirow{3}{*}{ Species } & \multirow{3}{*}{$N$} & \multicolumn{12}{|c|}{ Position } & \multirow{3}{*}{ Phylogeny } \\
\hline & & \multicolumn{3}{|c|}{ COI (sites 1407-1536) } & \multicolumn{9}{|c|}{ COII (sites 1-639) } & \\
\hline & & 1407 & 1485 & 1503 & 69 & 72 & 231 & 232 & 234 & 399 & 435 & 486 & 570 & \\
\hline D. $\operatorname{lin} i$ & 7 & $\mathrm{C}$ & $\mathrm{Y}$ & $\mathrm{T}$ & $\mathrm{T}$ & $\mathrm{C}$ & $\mathrm{C}$ & $\mathrm{T}$ & $\mathrm{R}$ & Y & W & A & $\mathrm{R}$ & Non-monophyletic \\
\hline D. ohnishii & 4 & $\mathrm{C}$ & $\mathrm{C}$ & $\mathrm{T}$ & $\mathrm{T}$ & $\mathrm{C}$ & $\mathrm{C}$ & Y & A & $\mathrm{T}$ & A & A & A & Non-monophyletic \\
\hline D. ogumai & 2 & Y & $\mathrm{T}$ & $\mathrm{C}^{*}$ & $\mathrm{C}^{*}$ & $\mathrm{~T}^{*}$ & $\mathrm{~T}^{*}$ & $\mathrm{~T}$ & A & $\mathrm{T}$ & A & $\mathrm{G}^{*}$ & A & Monophyletic \\
\hline
\end{tabular}

character-based approach failed to distinguish between the two non-monophyletic species for the COI-COII sequences as well.

Sequences of the ITS region covering a part of ITS1, the whole $5.8 \mathrm{~S}$ rDNA, ITS2a, 2S rDNA, and a part of ITS2 were amplified from 10 strains of $D$. lini and its siblings and some other species of the montium subgroup. The positions of nucleotides in the sequence were determined by alignment with the ITS sequence of $D$. simulans [26].
The GenBank accession numbers for these sequences are AY739939-AY739956. The 5.8S rDNA, ITS2a, and 2S rDNA were very conservative in all compared species, without variation in the sequence length. On the other hand, the ITS1 and ITS2 diverged largely in respect of either nucleotide substitution or sequence length. The NJ tree for the 10 strains of $D$. lini and its siblings showed the monophyly of $D$. ogumai but non-monophyly for either D. lini or D. ohnishii (Figure 3). Four informative sites were present in this region, 


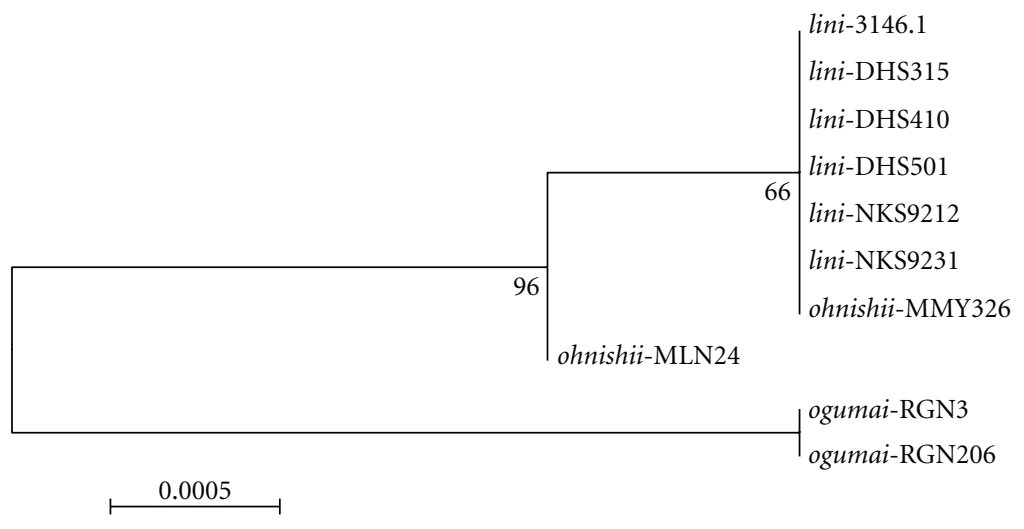

FIgURE 3: Neighbor-joining (NJ) tree inferred from ITS1-ITS2 sequences of ten strains of $D$. lini and its sibling species (D. ohnishii and $D$. ogumai). Numbers below branches indicate the bootstrap percentages.

TAble 5: Nucleotides at four informative sites in ITS1-ITS2 sequences of ten strains of $D$. lini and its sibling species (D. ohnishii and D. ogumai). Diagnostic nucleotides for DNA barcoding are indicated with an asterisk. The positions of nucleotide sites are based on the sequence of D. ogumai. Determination of each region in the sequence is based on the whole sequence of D. simulans (Z28413), ITS1: 690 bp; 5.8S: 123 bp; ITS2a: 26 bp; 2S: 30 bp; ITS2: 383 bp [26]. The partial or whole sequence of each region was obtained in D. ogumai as: ITS1: last 81 bp; 5.8S: 123 bp; ITS2a: 28 bp; 2S: 30 bp; ITS2: first 392 bp. See Table 3 for further explanations.

\begin{tabular}{|c|c|c|c|c|c|c|}
\hline \multicolumn{7}{|c|}{ Position } \\
\hline \multirow{2}{*}{ Species } & \multirow{2}{*}{$N$} & ITS1 (last $81 \mathrm{bp}$ ) & \multicolumn{3}{|c|}{ ITS2 (first 392 bp) } & \multirow{2}{*}{ Pylogeny } \\
\hline & & 16 & 307 & $312-325$ & 326 & \\
\hline D. lini & 6 & G & $\mathrm{T}$ & GTCAATAATAAAAT & - & Non-monophyletic \\
\hline D. ohnishii & 2 & G & $\mathrm{T}$ & GTCAATAATAAAAT/deletion & - & Non-monophyletic \\
\hline D. ogumai & 2 & $\mathrm{~A}^{*}$ & $\mathrm{G}^{*}$ & GTCAATAATAAAAT & $\mathrm{T}^{*}$ & Monophyletic \\
\hline
\end{tabular}

of which two nucleotide substitutions and one insertion were diagnostic for D. ogumai (Table 5); the remaining one, a 14-bp indel (sites 312-325) of ITS2, was polymorphic in $D$. ohnishii. For this region as well, no diagnostic nucleotide was found in either D. lini or D. ohnishii.

3.2. Molecular Phylogeny. The primary concordance tree (Figure 4) resulting from the Bayesian concordance analysis for the three loci (ND2, COI-COII, and ITS1-ITS2) was not discordant, especially the same for the strains of $D$. lini and its sibling species, in topology from the NJ tree (Figure 5) constructed using the concatenated sequences of the three regions (ND2 + COI-COII + ITS1-ITS2, 2442 bp in length), indicating that the mitochondrial and nuclear loci are concordant in the genealogies. The estimated divergence times based on a calibration point of 5.4 Mya divergence between $D$. melanogaster and D. simulans [35] were also shown in Figure 5. The three focal sibling species, D. lini, D. ohnishii, and D. ogumai, formed a monophyletic group supported by a high bootstrap value, $96 \%$. This clade (henceforth termed the lini clade) formed another, strongly supported $(100 \%)$ clade with $D$. kikkawai and its siblings, D. leontia and $D$. bocki, although the monophyly of the latter three sibling species was not supported. The relationships between the lini-kikkawai clade, D. barbarae (another species sampled from the kikkawai complex), and D. jambulina of the jambulina complex were not resolved. Within the lini clade, two distinct subclades, D. ogumai and D. lini+D. ohnishii, were recognized, with high support values, $100 \%$ and $98 \%$, respectively. Within the subclade of $D$. lini $+D$. ohnishii, either species did not form a monophyletic branch: the strain DHS315 of D. lini branched off first (bootstrap value 93\%), followed by the strain MMY326 of D. ohnishii (66\%), but there was no nucleotide variation in the concerned sequences among the rest strains including those of $D$. lini from Taiwan, Dinghushan (DHS) and Nankunshan (NKS) in Guangdong Province, and MLN24 of D. ohnishii from southern Yunnan.

The ancestor of the lini clade was estimated to have appeared about 2.23 Mya. Within the lini clade, the divergence between D. ogumai and D. lini was estimated to have first occurred $1.42 \mathrm{Mya}$, and then $D$. ohnishii was estimated to have speciated from $D$. lini very recently, at least after 0.17 Mya.

\section{Discussion}

In this study, we tested the applicability of DNA barcoding "species identification" to the lini clade consisting of three sibling species, which are morphologically almost indistinguishable [15] but have proved to be more or less reproductively isolated from one another $[16,18]$. We took two approaches, the phylogeny-based and character-based methods for DNA barcoding "species identification." However, neither method succeeded in identifying all these three species. The phylogeny-based method revealed the monophyly of D. ogumai and the character-based method found some 


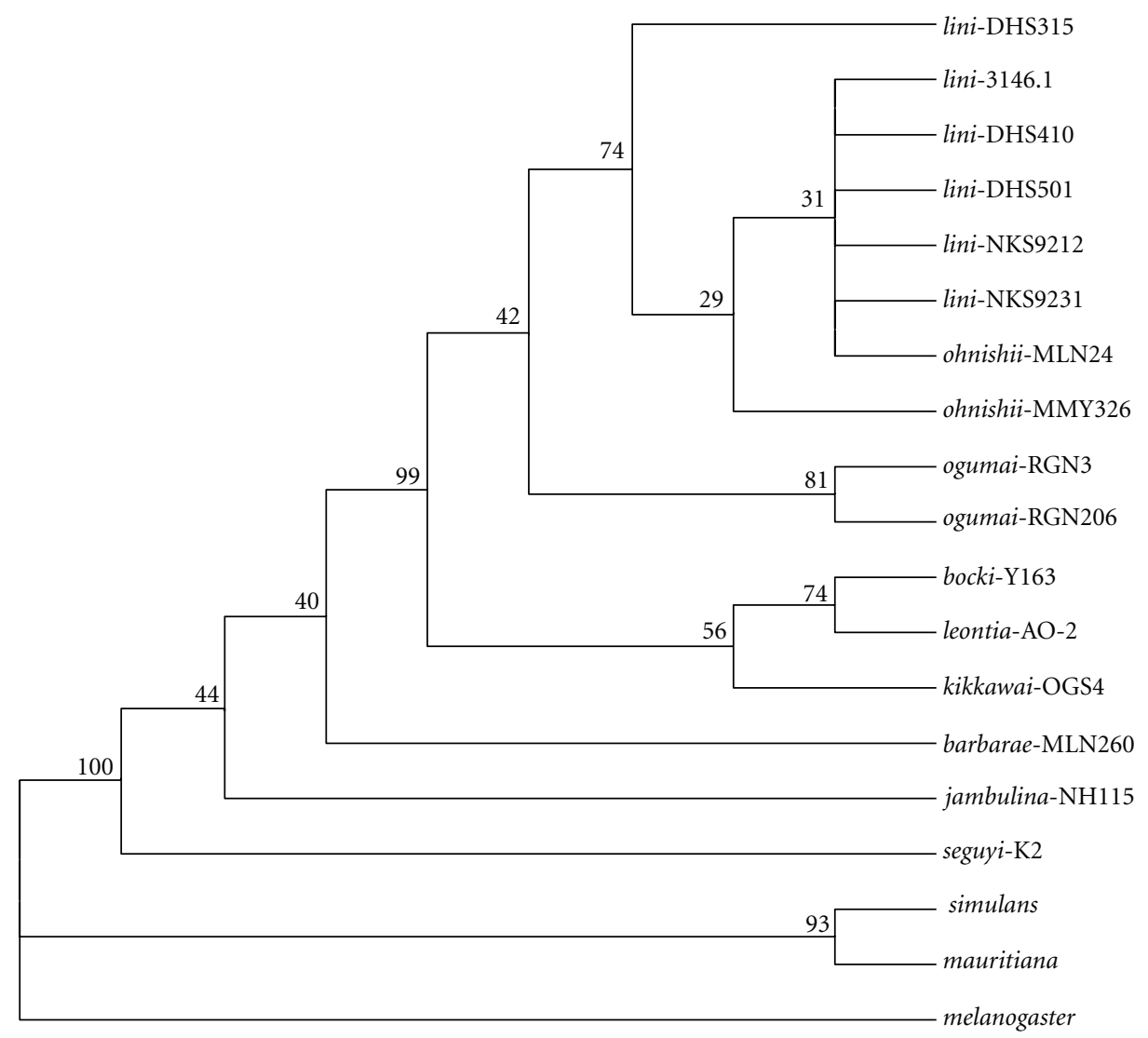

FIGURE 4: The primary concordance tree resulting from the Bayesian concordance analysis for the three regions, ND2, COI-COII, and ITS1ITS2. Numbers above branches are the concordance factors.

diagnostic nucleotides for D. ogumai, which can be, if not easily, distinguished from the other two species by a few morphological diagnostic characters [15].

However, we failed to get informative sites for DNA barcoding of two very closely related species, $D$. lini and D. ohnishii. This provides another evidential case that DNA barcoding is not always effective in species delineation, which has been corroborated in a number of cases of the genus Drosophila as a model system [40]. One possible problem is what genes are to be selected for DNA barcoding. Machado and Hey [41] pointed out that the well-established mutual monophyly of two closed related species, D. pseudoobscura and $D$. persimilis, was not recovered by phylogeny reconstruction based on nonrecombining molecules (particularly mitochondrial genome), but was strongly supported by that based on recombining molecules (five X-linked loci). The reason for the former is gene introgression between the species [41]. This may or may not be the case between $D$. lini and D. ohnishii as well. On the other hand, recombining molecules (inversions regions) may have contributed to the speciation process by affecting the hybrid fitness [42]. Socalled speciation genes involved in the pre- and postmating isolations might be good candidate genes for DNA barcoding and, of course, are very important to understand speciation mechanisms of such species at initial speciation. However, DNA barcoding based on such a standard marker as the 648- bp fragment of COI in the Barcode of Life project
$[11,12]$ should be a promising tool for nonexperts to easily and rapidly identify most of known species.

The inferred phylogenetic tree based on the concatenated sequences of the three regions did not support the monophyly of the kikkawai complex, although the taxon sampling was quite limited, covering only seven out of 12 species of this complex, in this study. Other studies, though under limited taxon sampling as well, suggested nonmonophyly of this species complex $[14,22,23,43]$. The delimitation of this species complex should be revised on the basis of molecular phylogenetic analyses under more comprehensive taxon sampling. However, the tree strongly supported the lini clade comprising the three sibling species, D. lini, D. ohnishii, and D. ogumai, and placed it close to D. kikkawai and its sibling species, in consistence with previous studies [14, 20-23].

With respect to the evolution of the lini clade, a hypothesis that the premating isolation has evolved through the process of reinforcement in the secondary contact zone between parapatric neighbors, D. ohnishii/D. lini or D. ohnishii/D. ogumai, has been proposed, since the premating isolation is absent between allopatric species, D. lini and D. ogumai [18]. Based on the phylogeny inferred from the present study and all available biological information from previous studies, we refine or revise the above hypothesis.

The ancestor of the lini clade should have derived as a close relative to D. kikkawai and/or its sibling species about 2.23 Mya presumably in the subtropics of the Oriental 


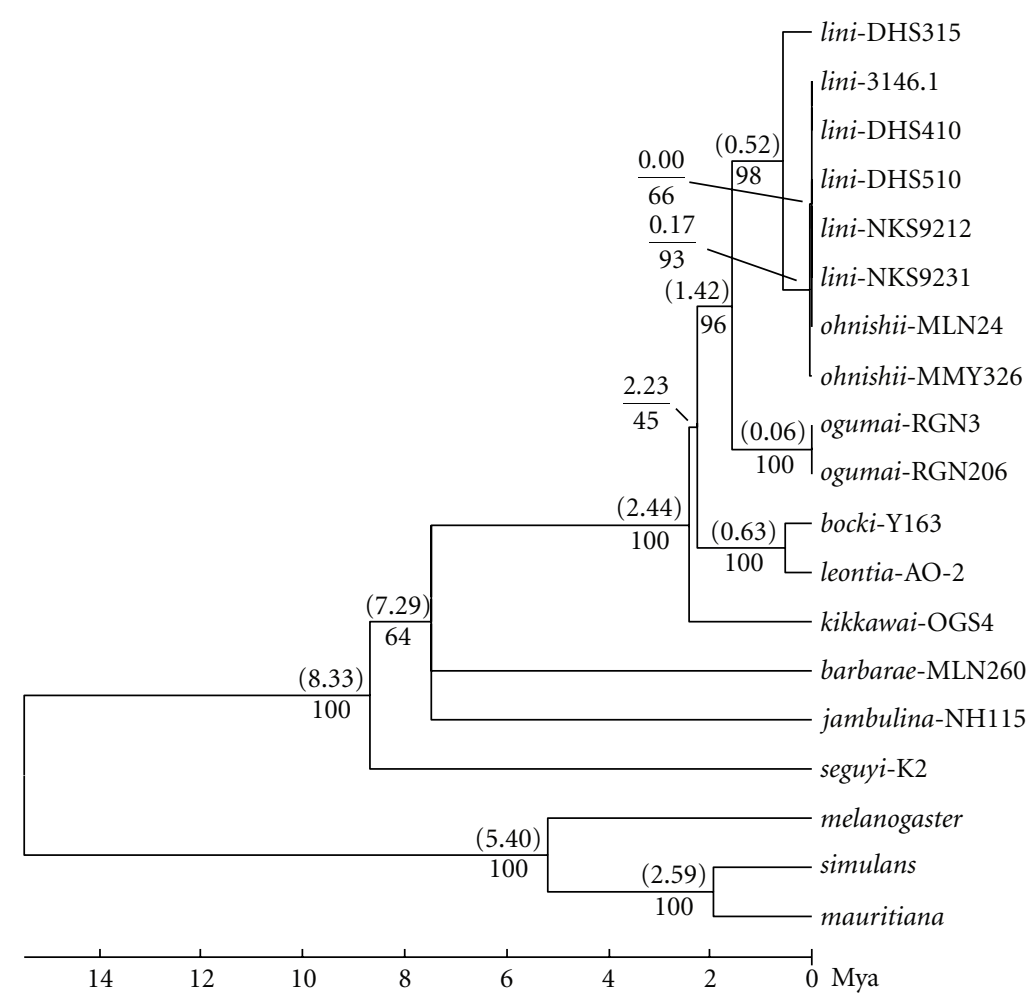

FIGURE 5: NJ tree inferred from the concatenated sequences (2442 bp) of three regions, ND2 (926 bp), COI-COII (842 bp), and ITS1-ITS2 (674 bp). The time scale (in Mya) was given to the tree on the basis of an estimated time, 5.4 Mya, for the divergence between $D$. melanogaster and D. simulans [35] as a calibration point. MEGA 5.05 [29] was used for constructing the tree (bootstrap test: 1000 replications; model: Kimura 2-parameter; gaps: treated by pairwise deletion). Numbers in parentheses above branches indicate divergence times (Mya), and those below branches bootstrap percentages.

Region. Then, the first speciation event producing D. ogumai and $D$. lini may have occurred about $1.42 \mathrm{Mya}$, and, finally, $D$. ohnishii may have diverged from $D$. lini very recently (at least after $0.17 \mathrm{Mya}$ ). This speciation order seems to be congruent with the morphological differentiation among the three species: D. ogumai can be distinguished from the other two species by a few diagnostic qualitative characters of the male genitalia and also is most remote from the other two species in terms of morphological distance based on metric characters [15]. In addition, variation in the strength of postmating isolation among the three species seems to be congruent as well with the speculated speciation order: the complete postmating isolation (production of no F1 hybrids) is present between the most diverged species, D. ogumai and D. ohnishii, while the postmating isolation is partial, producing F1 fertile female but sterile male hybrids, between the other pairs of species [16].

As a cue for the premating isolation caused by female repelling behavior, the frequency of sine song generated by males in their copulatory courtships has strongly been suggested from the evidence that it is different among the three species (significantly lower in D. ohnishii than in $D$. lini and D. ogumai) in accordance with the modes of sexual isolation between them [18]. Furthermore, wing-cut and playback experiments have provided crucial evidence for that the sine song frequency is used as a sexual cue for mate recognition in the lini clade $[44,45]$. In light of the inferred phylogeny, it is most parsimonious to consider that the lower frequency sine song has evolved in D. ohnishii. If so, the first speciation between D. ogumai and D. lini should have occurred allopatrically, because sympatric or parapatric speciation seems to be inconceivable under the absence of premating isolation. As for the second speciation of $D$. ohnishii from $D$. lini, two hypotheses can be conceived with respect to the evolutionary sequence of post- and premating isolations: (1) if the postmating isolation has first evolved, it should have been established between geographically isolated populations as in the first speciation event. Then, the premating isolation, that is, the lower frequency sine song, may have evolved through the process of reinforcement in the secondary contact zone with either neighboring species in D. ohnishii. Even in the light of the phylogeny inferred from the present study, we cannot determine the secondary contact to which species has promoted this evolution. (2) If the premating isolation has evolved first, the change in frequency of sine song has occurred and fixed in $D$. ohnishii as a consequence of adaptation to specific, but unknown, environmental conditions or as a neutral change irrespective of any adaptation and has secondarily come to function, actually or potentially, as a cue for mate recognition by females in this group. If this is the case, D. ohnishii would have speciated from a small local population, where 
such (a) mutant gene(s) causing differentiation of sexual character(s) are apt to be fixed, within the range of $D$. lini. However, the mtDNA haplotype polymorphism observed in D. ohnishii (Figures 1 and 2) seems to be inconsistent with this hypothesis, although it does not rule out the possibility of gene introgression after speciation, especially from southern China (DHS and NKS) populations of D. lini to southwestern China (MLN) population of D. ohnishii.

For the establishment of postmating isolation, another possibility is infection of microorganisms that cause cytoplasmic incompatibility [46-48]. However, Wolbachia infection has never been detected from any strains of the lini clade (M. Watada, personal communication). From another aspect, however, there remain large areas lacking distribution data between the ranges of the three species, especially between Xishuangbanna, southern Yunnan (the eastmost population of D. ohnishii) and Dinghushan, central Guangdong (the westmost population of $D$. lini). Filling this gap of data will prompt us to revise the hypothesis about the evolution of these two species seemingly having diverged very recently. In addition, studies of speciation genes relating to the postand premating isolations, especially those underlying the differentiation of sine song frequency, are needed.

\section{Acknowledgments}

This work was partly supported by Grants-in-Aid for Scientific Research from Japan Society for the Promotion of Science (nos. 12375002, 15255006, and 16370040) and the Grant of Natural Science Foundation of China (30970401). The authors thank Dr. T. Aotsuka for the material support and helpful discussion, and Dr. M. Watada for the information on Wolbachia infection in the strains of the Drosophila lini clade. S.-Y. Wen thanks Professor X.-F. Pang, a late Academician of the Chinese Academy of Sciences, for his spirit in science and education.

\section{References}

[1] Y. Satta and N. Takahata, "Evolution of Drosophila mitochondrial DNA and the history of the melanogaster subgroup," Proceedings of the National Academy of Sciences of the United States of America, vol. 87, no. 24, pp. 9558-9562, 1990.

[2] E. N. Moriyama and J. R. Powell, "Synonymous substitution rates in Drosophila: mitochondrial versus nuclear genes," Journal of Molecular Evolution, vol. 45, no. 4, pp. 378-391, 1997.

[3] R. M. Kliman, P. Andolfatto, J. A. Coyne et al., "The population genetics of the origin and divergence of the Drosophila simulans complex species," Genetics, vol. 156, no. 4, pp. 19131931, 2000.

[4] J. W. Ballard, "Comparative genomics of mitochondrial DNA in members of the Drosophila melanogaster subgroup," Journal of Molecular Evolution, vol. 51, no. 1, pp. 48-63, 2000.

[5] M. D. Dean and J. W. O. Ballard, "High divergence among Drosophila simulans mitochondrial haplogroups arose in midst of long term purifying selection," Molecular Phylogenetics \& Evolution, vol. 36, no. 2, pp. 328-337, 2005.

[6] C.-T. Ting, S.-C. Tsaur, S. Sun et al., "Gene duplication and speciation in Drosophila: evidence from the Odysseus locus,"
Proceedings of the National Academy of Sciences of the United States of America, vol. 101, no. 33, pp. 12232-12235, 2004.

[7] S. W. Schaeffer and E. L. Miller, "Nucleotide sequence analysis of Adh genes estimates the time of geographic isolation of the Bogota population of Drosophila pseudoobscura," Proceedings of the National Academy of Sciences of the United States of America, vol. 88, no. 14, pp. 6097-6101, 1991.

[8] R. L. Wang, J. Wakeley, and J. Hey, "Gene flow and natural selection in the origin of Drosophila pseudoobscura and close relatives," Genetics, vol. 147, no. 3, pp. 1091-1106, 1997.

[9] DrosWLD-Species, 2011, http://bioinfo.lowtem.hokudai.ac.jp/ $\mathrm{db} /$ modules/stdb/.

[10] M. Schiffer, M. E. Carew, and A. A. Hoffmann, "Molecular, morphological and behavioural data reveal the presence of a cryptic species in the widely studied Drosophila serrata species complex," Journal of Evolutionary Biology, vol. 17, no. 2, pp. 430-442, 2004.

[11] P. D. N. Hebert, A. Cywinska, S. L. Ball, and J. R. DeWaard, "Biological identifications through DNA barcodes," Proceedings of the Royal Society B, vol. 270, no. 1512, pp. 313-321, 2003.

[12] P. D. N. Hebert, S. Ratnasingham, and J. R. de Waard, "Barcoding animal life: cytochrome $\mathrm{c}$ oxidase subunit 1 divergences among closely related species," Proceedings of the Royal Society $B$, vol. 270, supplement 1, pp. S96-S99, 2003.

[13] U. Jinbo, T. Kato, and M. Ito, "Current progress in DNA barcoding and future implications for entomology," Entomological Science, vol. 14, no. 2, pp. 107-124, 2011.

[14] S. Ohnishi and T. K. Watanabe, "Systematics of the Drosophila montium species subgroup: a biochemical approach," Zoological Science, vol. 1, no. 5, pp. 801-807, 1984.

[15] M. T. Zannat and M. J. Toda, "Morphological comparison among Drosophila lini and its two new sibling species (Diptera: Drosophilidae)," Zoological Science, vol. 19, no. 12, pp. 13771388, 2002.

[16] Y. Oguma, S. Wen, M. Tomaru, H. Matsubayashi, and T. Peng, "Reproductive isolation between Drosophila lini and its siblings," The Japanese Journal of Genetics, vol. 70, no. 3, pp. 311-320, 1995.

[17] B. K. Kim, T. K. Watanabe, and O. Kitagawa, "Evolutionary genetics of the Drosophila montium subgroup. I. Reproductive isolations and the phylogeny," The Japanese Journal of Genetics, vol. 64, no. 3, pp. 177-190, 1989.

[18] S.-Y. Wen, H. Yamada, Y.-F. Li et al., "Copulatory courtship behavior and sine song as a mate recognition cue in Drosophila lini and its sibling species," Zoological Science, vol. 28, no. 7, pp. 469-475, 2011.

[19] L. Tsacas and J. David, "Systematics and biogeography of the Drosophila kikkawai complex, with descriptions of new species (Diptera, Drosophilidae)," Annales de la Société entomologique de France, vol. 13, pp. 675-693, 1977.

[20] S. Ohnishi, K. W. Kim, and T. K. Watanabe, "Biochemical phylogeny of the Drosophila montium species subgroup," The Japanese Journal of Genetics, vol. 58, pp. 141-151, 1983.

[21] B. K. Kim, T. Aotsuka, and O. Kitagawa, "Evolutionary genetics of the Drosophila montium subgroup. II. Mitochondrial DNA variation," Zoological Science, vol. 10, pp. 991-996, 1993.

[22] V. Schawaroch, "Phylogeny of a paradigm lineage: the Drosophila melanogaster species group (Diptera: Drosophilidae)," Biological Journal of the Linnean Society, vol. 76, no. 1, pp. 2137, 2002.

[23] J.-L. Da Lage, G. J. Kergoat, F. Maczkowiak, J. F. Silvain, M.-L. Cariou, and D. Lachaise, "A phylogeny of Drosophilidae using the Amyrel gene: questioning the Drosophila melanogaster 
species group boundaries," Journal of Zoological Systematics and Evolutionary Research, vol. 45, no. 1, pp. 47-63, 2007.

[24] K. van der Linde, D. Houle, G. S. Spicer, and S. J. Steppan, "A supermatrix-based molecular phylogeny of the family Drosophilidae," Genetics Research, vol. 92, no. 1, pp. 25-38, 2010.

[25] C. Simon, "Molecular systematics at the species boundary: exploiting conserved and variable regions of the mitochondrial genome of animals via direct sequencing from amplified DNA," in Molecular Techniques in Taxonomy, G. M. Hewitt, A. W. B. Johnston, and J. P. W. Young, Eds., pp. 33-71, Springer, Heidelberg, Germany, 1991.

[26] C. Schlotterer, M. T. Hauser, A. von Haeseler, and D. Tautz, "Comparative evolutionary analysis of rDNA ITS regions in Drosophila," Molecular Biology and Evolution, vol. 11, no. 3, pp. 513-522, 1994.

[27] S. Y. Wen, Studies on the speciation of Drosophila lini and its related species, Ph.D. thesis, South China Agricultural University, 2004.

[28] S. Y. Wen and X. F. He, "Rapid preparation of trace-DNA templet from insects and other animals for PCR," Entomological Knowledge, vol. 40, pp. 110-113, 2003.

[29] K. Tamura, D. Peterson, N. Peterson, G. Stecher, M. Nei, and S. Kumar, "MEGA5: Molecular Evolutionary Genetics Analysis using maximum likelihood, evolutionary distance, and maximum parsimony methods," Molecular Biology and Evolution, vol. 28, no. 10, pp. 2731-2739, 2011.

[30] Z. Abdo and G. B. Golding, "A step toward barcoding life: a model-based, decision-theoretic method to assign genes to preexisting species groups," Systematic Biology, vol. 56, no. 1, pp. 44-56, 2007.

[31] K. Munch, W. Boomsma, J. P. Huelsenbeck, E. Willerslev, and R. Nielsen, "Statistical assignment of DNA sequences using Bayesian phylogenetics," Systematic Biology, vol. 57, no. 5, pp. 750-757, 2008.

[32] K. Munch, W. Boomsma, E. Willerslev, and R. Nielsen, "Fast phylogenetic DNA barcoding," Philosophical Transactions of the Royal Society B, vol. 363, no. 1512, pp. 3997-4002, 2008.

[33] M. Lou and G. Brian Golding, "Assigning sequences to species in the absence of large interspecific differences," Molecular Phylogenetics \& Evolution, vol. 56, no. 1, pp. 187-194, 2010.

[34] R. DeSalle, M. G. Egan, and M. Siddall, "The unholy trini- ty: taxonomy, species delimitation and DNA barcoding," Philosophical Transactions of the Royal Society B, vol. 360, no. 1462, pp. 1905-1916, 2005.

[35] K. Tamura, S. Subramanian, and S. Kumar, “Temporal patterns of fruit fly (Drosophila) evolution revealed by mutation clocks," Molecular Biology and Evolution, vol. 21, no. 1, pp. 3644, 2004.

[36] C. Ané, B. Larget, D. A. Baum, S. D. Smith, and A. Rokas, "Bayesian estimation of concordance among gene trees," Molecular Biology and Evolution, vol. 24, no. 2, pp. 412-426, 2007.

[37] J. P. Huelsenbeck and F. Ronquist, "MRBAYES: Bayesian inference of phylogenetic trees," Bioinformatics, vol. 17, no. 8, pp. 754-755, 2001.

[38] J. J. Gao, H. A. Watabe, T. Aotsuka, J. F. Pang, and Y. P. Zhang, "Molecular phylogeny of the Drosophila obscura species group, with emphasis on the Old World species," BMC Evolutionary Biology, vol. 7, article 87, 2007.

[39] D. O. Clary and D. R. Wolstenholme, "The mitochondrial DNA molecule of Drosophila yakuba: nucleotide sequence, gene organization, and genetic code," Journal of Molecular Evolution, vol. 22, no. 3, pp. 252-271, 1985.

[40] A. Yassin, T. A. Markow, A. Narechania, P. M. O'Grady, and R. DeSalle, "The genus Drosophila as a model for testing tree- and character-based methods of species identification using DNA barcoding," Molecular Phylogenetics \& Evolution, vol. 57, no. 2, pp. 509-517, 2010.

[41] C. A. Machado and J. Hey, "The causes of phylogenetic conflict in a classic Drosophila species group," Proceedings of the Royal Society B, vol. 270, no. 1520, pp. 1193-1202, 2003.

[42] M. A. F. Noor, K. L. Gratos, L. A. Bertucci, and J. Reiland, "Chromosomal inversions and the reproductive isolation of species," Proceedings of the National Academy of Sciences of the United States of America, vol. 98, no. 21, pp. 12084-12088, 2001.

[43] Y. Yang, Y. P. Zhang, Y. H. Qian, and Q. T. Zeng, "Phylogenetic relationships of Drosophila melanogaster species group deduced from spacer regions of histone gene H2A-H2B," Molecular Phylogenetics \& Evolution, vol. 30, no. 2, pp. 336-343, 2004.

[44] Y. F. Li, The role of sine song in copulatory courtship and its genetic control in three species of Drosophila montium subgroup, Ph.D. thesis, South China Agricultural University, 2011.

[45] Y. F. Li, S. Y. Wen, and M. G. Ritchie, "Copulatory song in three species of the Drosophila montium subgroup extends copulation and shows unusual genetic control," Animal Behaviour, vol. 83, pp. 233-238, 2012.

[46] J. A. J. Breeuwer and J. H. Werren, "Microorganisms associated with chromosome destruction and reproductive isolation between two insect species," Nature, vol. 346, no. 6284, pp. 558$560,1990$.

[47] S. L. O’Neill and T. L. Karr, "Bidirectional incompatibility between specific populations of Drosophila simulans," Nature, vol. 348, no. 6297, pp. 178-180, 1990.

[48] J. W. O. Ballard and A. C. James, "Differential fitness of mitochondrial DNA in perturbation cage studies correlates with global abundance and population history in Drosophila simulans," Proceedings of the Royal Society B, vol. 271, no. 1544, pp. 1197-1201, 2004. 

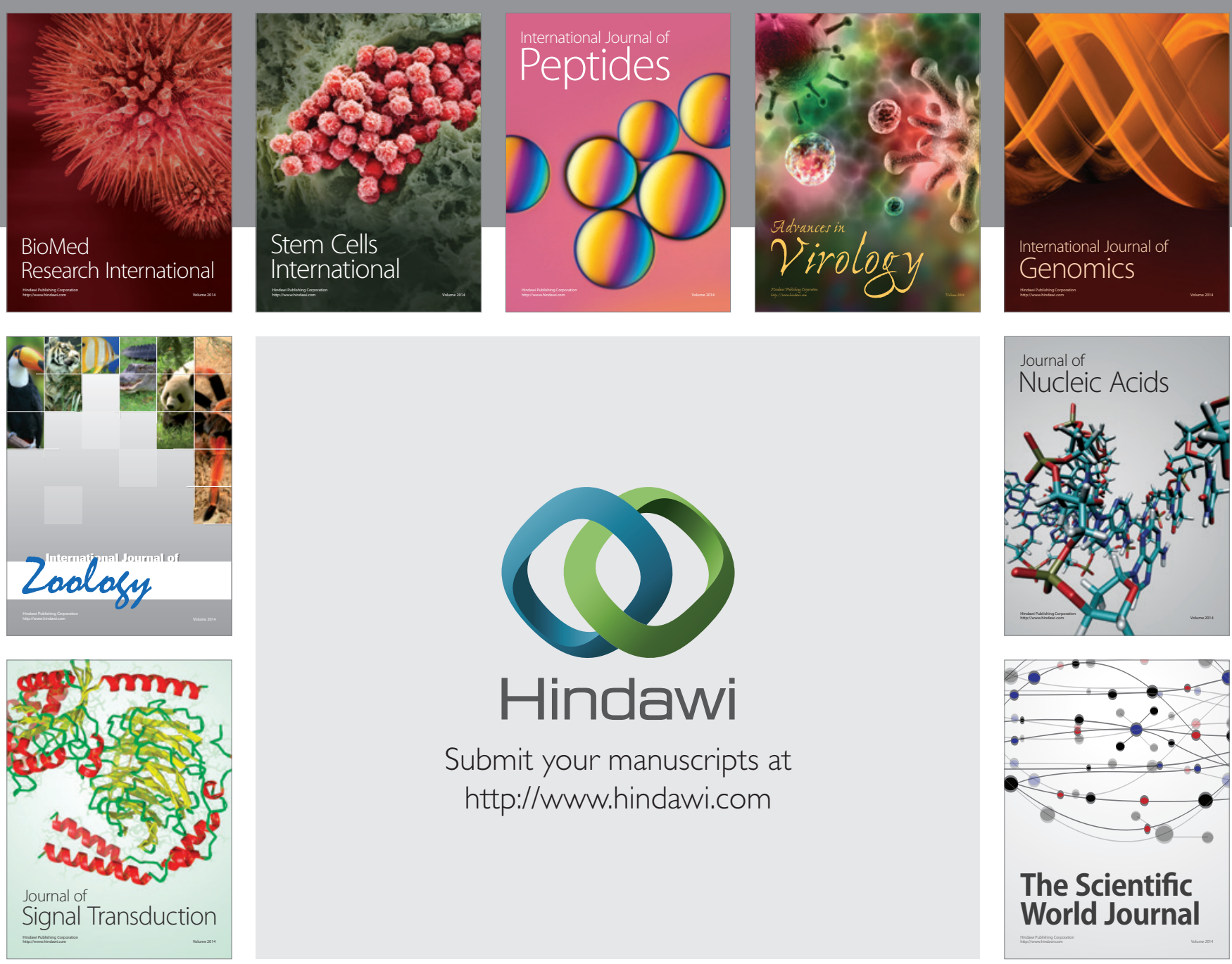

Submit your manuscripts at

http://www.hindawi.com
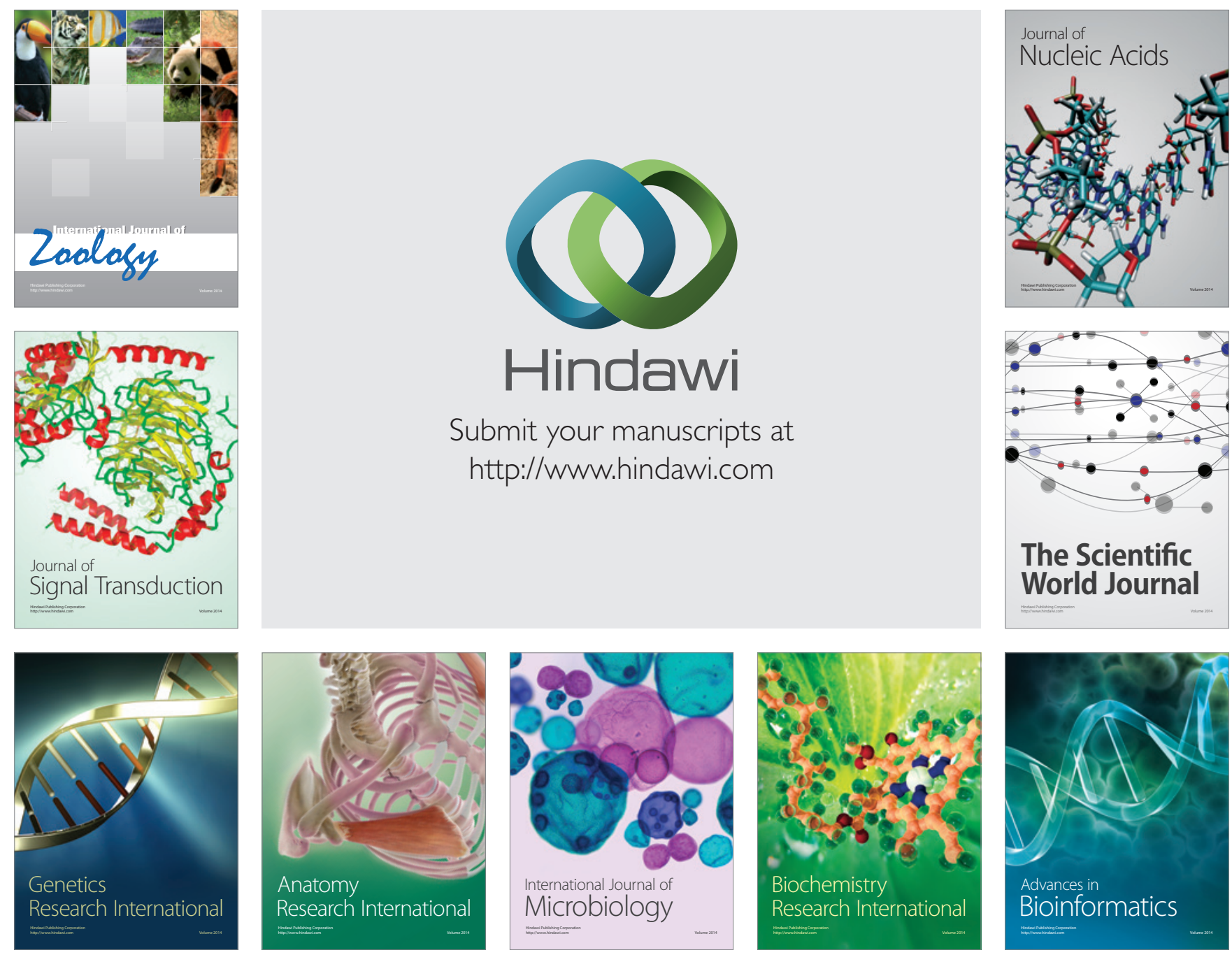

The Scientific World Journal
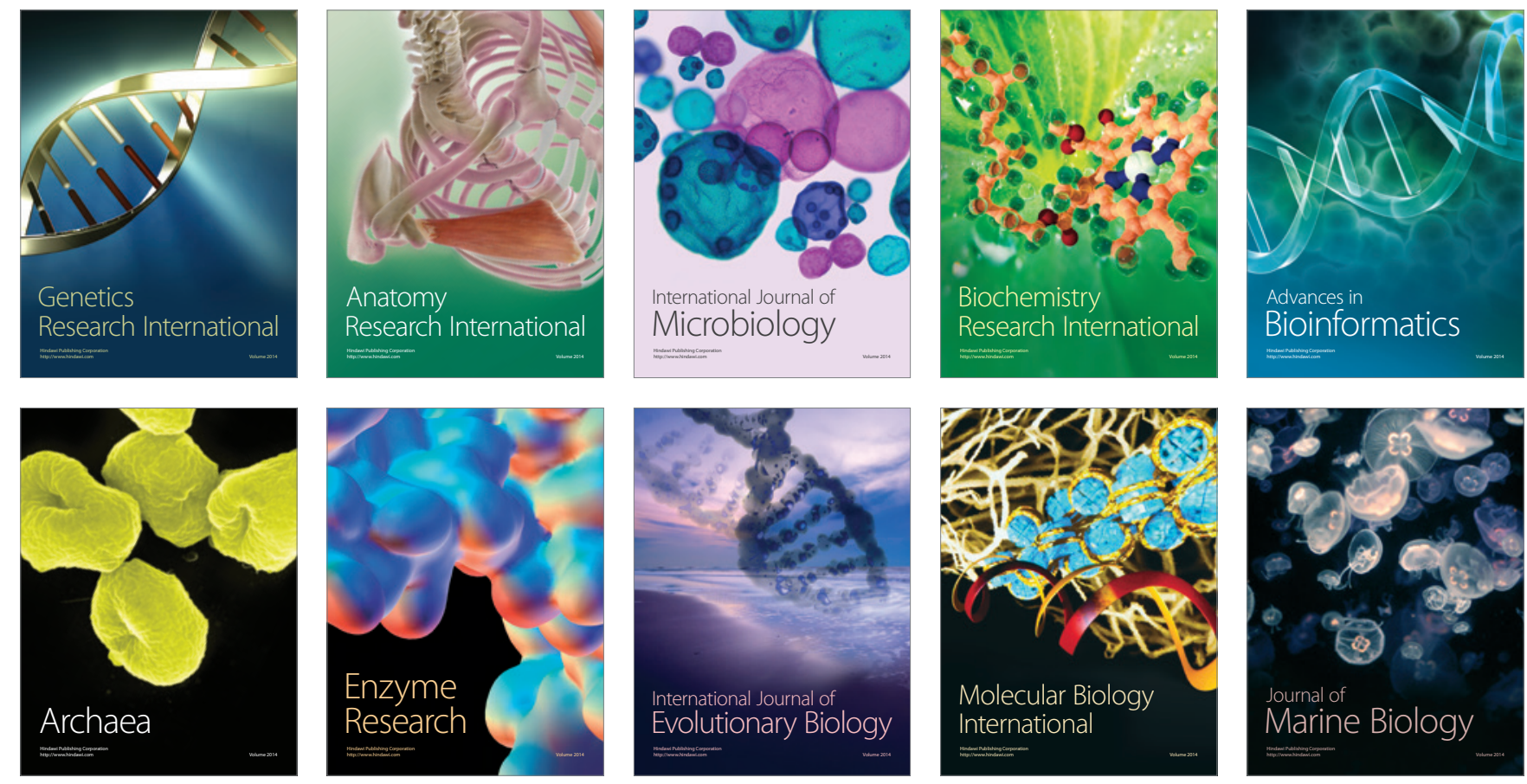